\title{
Sodium-Glucose Cotransporter-2 (SGLT-2) Attenuates Serum Uric Acid (SUA) Level in Patients with Type 2 Diabetes
}

\author{
Mazhar Hussain $\mathbb{D}^{1},{ }^{1}$ Asim Elahi, ${ }^{2}$ Abid Hussain, ${ }^{3}$ Javed Iqbal, ${ }^{4}$ Lubna Akhtar, ${ }^{1}$ \\ and Abdul Majid ${ }^{5}$ \\ ${ }^{1}$ Department of Pharmacology \& Therapeutics Sheikh Zayed Medical College/Hospital, Rahim Yar Khan, Punjab, Pakistan \\ ${ }^{2}$ Department of Medicine CHI Saint Joseph Health Hospital, London, Kentucky, USA \\ ${ }^{3}$ Department of Nephrology Sheikh Zayed Medical College/Hospital, Rahim Yar Khan, Pakistan \\ ${ }^{4}$ Department of Medicine Sheikh Zayed Medical College/Hospital, Rahim Yar Khan, Pakistan \\ ${ }^{5}$ Department of Cardiology, Sheikh Zayed Medical College \& Hospital Rahim Yar Khan, Pakistan
}

Correspondence should be addressed to Mazhar Hussain; mazharhussain214@gmail.com

Received 4 April 2021; Revised 15 May 2021; Accepted 8 June 2021; Published 19 June 2021

Academic Editor: Claudia Cardoso

Copyright (c) 2021 Mazhar Hussain et al. This is an open access article distributed under the Creative Commons Attribution License, which permits unrestricted use, distribution, and reproduction in any medium, provided the original work is properly cited.

\begin{abstract}
Background. Hyperuricemia has a strong association with diabetes mellitus. Hyperuricemia can lead to cardiovascular and renal complications in patients with diabetes. The goal of this study was to compare the effect of sodium-glucose cotransporter-2 (SGLT-2) inhibitors dapagliflozin and empagliflozin on serum uric acid (SUA) levels in patients with type 2 diabetes against traditional oral antihyperglycemic drugs (OADs). Methods. In this double-blind randomized controlled trial, 70 patients with type 2 diabetes and elevated SUA levels were assigned to two treatment groups. Patients in group A received SGLT-2 inhibitors tablet dapagliflozin $5 \mathrm{mg}$ to $10 \mathrm{mg}$ and empagliflozin $10 \mathrm{mg}$ to $25 \mathrm{mg}$. Group B patients received OADs such as glimepiride, metformin, sitagliptin, gliclazide, and glibenclamide as monotherapy or combination therapy. The changes in SUA level were primary end points while changes in body weight and body mass index (BMI) from baseline to end point were secondary end points. Results. After four weeks of treatment, we noted a significant reduction of mean SUA levels in the SGLT-2 inhibitor group from $7.5 \pm 2.5$ to $6.3 \pm 0.8 \mathrm{mg} / \mathrm{dl}$ versus comparator group from $7.1 \pm 1.8$ to $6.8 \pm 2.2 \mathrm{mg} / \mathrm{dl}(p=0.001)$. Mean body weight was significantly reduced in the SGLT-2 group from $82 \pm 10.4$ to $78 \pm 12.5 \mathrm{~kg}$ versus comparator group from $78 \pm 13.2$ to $79.2 \pm 9.7 \mathrm{~kg}(p=0.001)$. Similarly, the mean BMI of patients in the SGLT-2 group was significantly reduced from $25.7 \pm 3.2$ to $24.2 \pm 3.2 \mathrm{~kg} / \mathrm{m}^{2}$ versus comparator group from $27.5 \pm 4.2$ to $28 \pm 3.6 \mathrm{~kg} / \mathrm{m}^{2}(p=0.002)$. Conclusion. SGLT-2 inhibitors have a strong potential to decrease SUA levels in patients with type 2 diabetes.
\end{abstract}

\section{Introduction}

The prevalence of diabetes is growing worldwide and continues to represent a global health challenge. In 2015, the estimated prevalence of diabetes was $8.8 \%$, but projections estimate this will rise to $10.4 \%$ by 2040 [1].The International Diabetes Federation estimates that the number of patients with diabetes will reach 552 million in 2030, adding significant morbidity and mortality for patients and social and economic burdens on health systems. Identifying and preventing risk factors for diabetes are urgent to stop the diabetes epidemic [2].
Hyperuricemia is a disorder of purine metabolism. Serum uric acid (SUA) levels are usually elevated in patients with diabetes compared to healthy individuals. Hyperuricemia increases the risk of diabetes in people who have impaired glucose tolerance, and hyperuricemia is highly prevalent in patients with diabetes $[3,4]$. SUA is a strong independent, novel risk factor for diabetes [5]. Increases in body weight, waist circumference, dyslipidemia, sedentary lifestyle, hypertension, and insulin resistance are predisposing factors for hyperuricemia in patients with diabetes $[6,7]$.

Hyperuricemia has a strong association with type 2 diabetes and is a strong predictor for developing metabolic 
syndrome, diabetes mellitus, and hypertension. Hyperuricemia leads to significant morbidity and mortality in the form of kidney and cardiovascular diseases. Reducing SUA in patients with type 2 diabetes may reduce micro and macro complications [8-10].

Sodium-glucose cotransporter-2 (SGLT-2) inhibitors comprise a novel class of oral antihyperglycemic drugs (OADs) with insulin-independent effects. The unique mechanism of SGLT-2 inhibitors is beneficial to patients with diabetes by inhibiting glucose reabsorption from the proximal tubule of the kidney. SGLT-2 inhibitors have shown promising effects on body weight, blood pressure, dyslipidemia, and inflammation. Given their beneficial effects on the cardiovascular system and good safety profile, SGLT-2 inhibitors are now widely prescribed worldwide $[11,12]$.

Uricosuric and synthesis inhibitors are the two main drug groups used for the treatment of hyperuricemia. Although uricosuric drugs such as probenecid and losartan act through different urate transporters (e.g., URAT1) than SGLT-2 inhibitors (which affect urate transporter SLC2A9 isoform b), the net effect is the same: both drugs cause an increase in the excretion of uric acid in urine [13]. Some studies have shown that SGLT-2 inhibitors have a strong potential to reduce SUA levels.

Therefore, the present study was conducted to determine SGLT-2 inhibitor's effect on SUA levels after four weeks of treatment in patients with type 2 diabetes.

\section{Materials and Methods}

We conducted this four-week double-blind randomized controlled trial at a government clinic and five private clinics in the District of Rahim Yar Khan, South Punjab, Pakistan, from February to March 2020. Three hundred eighty overweight patients with type 2 diabetes on OAD agents were screened for elevated SUA levels. Of this group, 70 patients were enrolled in the study according to our inclusion and exclusion criteria. To be included in the study, patients must have a body mass index $(\mathrm{BMI})<28 \mathrm{~kg} / \mathrm{m}^{2}$, SUA levels $(>7.0 \mathrm{mg} / \mathrm{dl}$ for males and $>6.0 \mathrm{mg} / \mathrm{dl}$ for females) with adequate glycemic control (defined as glycated hemoglobin [HbAlc] $<7 \%$ ), and blood pressure within the reference range $(<140 / 90 \mathrm{mmHg})$. Patients with a history of smoking, alcohol use, and cardiovascular and renal disease were excluded from the study. Also, a complete history was taken to screen for drugs that can affect uric acid levels, such as thiazide diuretics, aspirin, niacin, statins, anticancer agents, immunosuppressants, and pyrazinamide. Patients taking any antigout drug were also excluded from the study. Patients were also asked to avoid all fructose-containing beverages and foods for the duration of the study.

Patients were randomly assigned to one of two groups by simple random number generation via computer software. Patients in group A $(n=35)$ were treated with daily empagliflozin tablet $10 \mathrm{mg}$ to $25 \mathrm{mg}$ and dapagliflozin $5 \mathrm{mg}$ to $10 \mathrm{mg}$, and patients in group $\mathrm{B}(n=35)$ continue their routine OADs either alone or in combination as the standard treatment of diabetes. These OADs were glimepiride, metformin, sitagliptin, gliclazide, and glibenclamide. Dosages of the drugs were adjusted according to patient fasting blood glucose and random blood glucose level testing. Patients, investigators, and laboratory staff were blinded to the study plan throughout clinical trial.

The changes in SUA level were primary end points while changes in body weight and body mass index (BMI) from baseline to end point were secondary end points.

All clinical and laboratory parameters were measured by following standard guidelines and methods. The study design was approved by the institutional review board of Sheikh Zayed Medical College/Hospital Rahim Yar Khan ( 26/IRB/SZMC/SZH). All study participants provided written informed consent.

\section{Data Analysis}

Values were expressed as mean \pm standard deviation and percentages. Numeric data were analyzed using SPSS Statistics for Windows, Version 18.0. (Chicago: SPSS Inc.). A $t$-test was used to compare the changes between the test groups. A paired $t$-test was applied to compare each group's changes, while the Mann-Whitney $U$-test and $t$-test were applied to compare changes between groups from baseline to four weeks. A $p$ value $<0.05$ was considered statistically significant.

\section{Results}

All patients completed the study with none lost to follow-up. In group A, 20 received empagliflozin while 15 patients received dapagliflozin. In group $B, 35$ patients received their routine OADs. The safety and tolerability profile of empagliflozin and dapagliflozin were quite good, and no adverse effects were recorded during the study.

The baseline demographic characteristics of study participants are presented in Table 1. SUA levels were higher in both groups at the start of the study. Serum uric acid levels significantly decreased in the SGLT-2 inhibitor group from $7.5 \pm 2.5 \mathrm{mg} / \mathrm{dl}$ to $6.3 \pm 0.8 \mathrm{mg} / \mathrm{dl}$, while the control group's SUA levels went from $7.1 \pm 1.8 \mathrm{mg} / \mathrm{dl}$ at baseline to $6.8 \pm$ $2.2 \mathrm{mg} / \mathrm{dl}$ by the end of the study period ( $p=0.001$; Table 2 ).

Mean body weight was significantly reduced in the SGLT-2 group from $82 \pm 10.4 \mathrm{~kg}$ to $78 \pm 12.5 \mathrm{~kg}$ compared to the comparator group, which had a mean body weight of $78 \pm 13.2 \mathrm{~kg}$ at baseline and $79.2 \pm 9.7 \mathrm{~kg}$ at the end of the study period $(p=0.001)$. Similarly, BMI was reduced significantly from $25.7 \pm 3.2 \mathrm{~kg} / \mathrm{m}^{2}$ to $24.2 \pm 3.2 \mathrm{~kg} / \mathrm{m}^{2}$ in the SGLT-2 inhibitor group while the control group's mean BMI at baseline was $27.5 \pm 4.2 \mathrm{~kg} / \mathrm{m}^{2}$ and $28 \pm 3.6 \mathrm{~kg} / \mathrm{m}^{2}$ by the end of the study $(p=0.002)$.

\section{Discussion}

To the best of our knowledge, this represents the first study conducted in Pakistan to determine the four-week effect of SGLT-2 inhibitors (dapagliflozin and empagliflozin) on SUA in patients with type 2 diabetes. Both drugs significantly reduced SUA as compared to control with good safety and tolerability profile. 
TABle 1: Baseline demographics characteristics $(N=70)$.

\begin{tabular}{|c|c|c|c|}
\hline Baseline parameters & 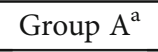 & Group Bb & $p$ value \\
\hline Age in years $($ mean $\pm S D)$ & $46 \pm 11.5$ & $43 \pm 13.2$ & 0.629 \\
\hline Male, $n(\%)$ & $24(68.5 \%)$ & $20(57 \%)$ & 0.170 \\
\hline Female, $n(\%)$ & $11(31.1 \%)$ & $15(42.8 \%)$ & 0.23 \\
\hline Duration of diabetes in years (mean $\pm S D$ ) & $8.2 \pm 4.8$ & $9.2 \pm 6.2$ & 0.236 \\
\hline Body weight in $\mathrm{kg}($ mean $\pm \mathrm{SD})$ & $82 \pm 10.4$ & $78 \pm 13.2$ & 0.324 \\
\hline $\mathrm{BMI}$ in $\mathrm{kg} / \mathrm{m}^{2}($ mean $\pm \mathrm{SD})$ & $25.7 \pm 3.2$ & $27.5 \pm 4.2$ & 0.763 \\
\hline Blood pressure systolic in $\mathrm{mmHg}($ mean $\pm \mathrm{SD})$ & $115 \pm 10.8$ & $125 \pm 8.5$ & 0.671 \\
\hline Blood pressure diastolic $\mathrm{mmHg}($ mean $\pm \mathrm{SD})$ & $80 \pm 4.8$ & $88 \pm 3.2$ & 0.162 \\
\hline Fasting blood glucose in $\mathrm{mg} / \mathrm{dl}($ mean $\pm \mathrm{SD})$ & $99 \pm 12.7$ & $105 \pm 10$ & 0.68 \\
\hline $\mathrm{HbAlc}($ mean $\pm \mathrm{SD})$ & $6.5 \pm 3.2$ & $7.0 \pm 2.8$ & 0.682 \\
\hline Serum uric acid level in $\mathrm{mg} / \mathrm{dl}($ mean $\pm \mathrm{SD})$ & $7.5 \pm 2.5$ & $7.1 \pm 1.8$ & 0.36 \\
\hline
\end{tabular}

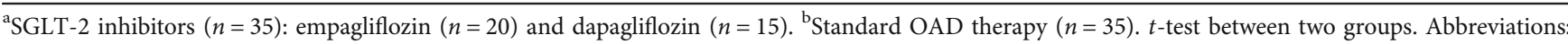
BMI: body mass index; HbAlc: glycated hemoglobin; OAD: oral antihyperglycemic drug; SD: standard deviation; SGLT-2: sodium-glucose cotransporter-2.

TABLE 2: Comparison of uric acid changes at baseline and after treatment.

\begin{tabular}{|c|c|c|c|c|c|c|c|}
\hline \multirow{2}{*}{ Parameters } & \multicolumn{2}{|c|}{ Group $A^{a}$} & \multirow{2}{*}{$p$ value $^{\mathrm{b}}$} & \multicolumn{2}{|c|}{ Group $B^{c}$} & \multirow{2}{*}{$p$ value $^{\mathrm{b}}$} & \multirow{2}{*}{$p$ value $^{\mathrm{d}}$} \\
\hline & Baseline & End point & & Baseline & End point & & \\
\hline Body weight in $\mathrm{kg}($ mean $\pm \mathrm{SD})$ & $82 \pm 10.4$ & $78 \pm 12.5$ & 0.005 & $78 \pm 13.2$ & $79.2 \pm 9.7$ & 0.02 & 0.001 \\
\hline $\mathrm{BMI}$ in $\mathrm{kg} / \mathrm{m}^{2}($ mean $\pm \mathrm{SD})$ & $25.7 \pm 3.2$ & $24.2 \pm 3.2$ & 0.001 & $27.5 \pm 4.2$ & $28 \pm 3.6$ & 0.056 & 0.002 \\
\hline Fasting blood glucose in $\mathrm{mg} / \mathrm{dl}($ mean $\pm \mathrm{SD})$ & $99 \pm 12.7$ & $90 \pm 10.2$ & 0.42 & $105 \pm 10$ & $95.5 \pm 10$ & 0.042 & 0.62 \\
\hline Serum uric acid level in $\mathrm{mg} / \mathrm{dl}($ mean $\pm \mathrm{SD})$ & $7.5 \pm 2.5$ & $6.3 \pm 0.8$ & 0.001 & $7.1 \pm 1.8$ & $6.8 \pm 2.2$ & 0.042 & 0.001 \\
\hline
\end{tabular}

${ }^{\mathrm{a}}$ SGLT-2 inhibitors $(n=35)$ : empagliflozin $(n=20)$ and dapagliflozin $(n=15) .{ }^{\mathrm{b}}$ Comparison within groups. ${ }^{\mathrm{c}}$ Standard OAD therapy $(n=35)$. ${ }^{\mathrm{d}}$ Comparison of changes of each variable between the two groups. Abbreviations: BMI: body mass index; HbAlc: glycated hemoglobin; OAD: oral antihyperglycemic drug; SD: standard deviation; SGLT-2: sodium-glucose cotransporter-2.

SGLT-2 inhibitors are widely prescribed for patients with diabetes as monotherapy and combination therapy. Their mechanism of action is unique among conventional OADs. They have an insulin-independent effect and increase the excretion of glucose in the urine. These drugs also have beneficial effects on conventional risk factors of cardiovascular disease $[14,15]$.

SGLT-2 inhibitors also lower SUA levels in various clinical studies [11, 13]. The only US Food and Drug Administration-approved SGLT-2 inhibitors are canagliflozin, dapagliflozin, empagliflozin, and ertugliflozin. Among those, dapagliflozin and empagliflozin are available in Pakistan. A study by Hao et al. [16] concluded that dapagliflozin significantly reduced SUA levels in patients with uncontrolled diabetes who were hospitalized over 24 hours. We noted similar results, but participants in this study have adequate glycemic control, and our study duration was four weeks.

Our results align with a meta-analysis that showed dapagliflozin significantly reduced SUA by $1.14 \mathrm{mg} / \mathrm{dl}$ at a dose of $10 \mathrm{mg}$ daily. However, in our study, both dapagliflozin and empagliflozin reduced uric acid by $1.21 \mathrm{mg} / \mathrm{dl}$ at different doses. We did not analyze the dose-dependent effects of the SGLT-2 inhibitors. Moreover, in these studies, the status regarding drugs affecting SUA was lacking. Our study excluded all patients receiving drugs that would affect SUA levels $[17,18]$.

A meta-analysis of 62 studies by Zhao et al. [18] concluded that empagliflozin reduced SUA more significantly than dapagliflozin. However, dapagliflozin showed dosedependent uric acid-lowering effects. In our study, both drugs significantly reduced SUA levels. Also, our study did not compare dapagliflozin against empagliflozin in changes to SUA level. Similarly, a systematic review and metaanalysis of 12 randomized trials over a follow-up period of $28 \pm 22$ weeks on empagliflozin $(10 \mathrm{mg}$ or $25 \mathrm{mg}$ ) revealed that it significantly reduced blood pressure, body weight, $\mathrm{HbAlc}$, and SUA [19].

A meta-analysis of 31 studies on 13,650 diabetic patients demonstrated that SGLT-2 inhibitors significantly decreased SUA levels compared with placebo, canagliflozin $0.62 \mathrm{mg} / \mathrm{dl}$, dapagliflozin $0.64 \mathrm{mg} / \mathrm{dl}$, and empagliflozin $0.71 \mathrm{mg} / \mathrm{dl}$ [20]. Our study showed a greater reduction of SUA by $1.21 \mathrm{mg} / \mathrm{dl}$, likely because we excluded all risk factors and drugs that can affect SUA levels.

The proposed mechanisms by which SGLT-2 inhibitors decrease SUA include the expression of glucose transporter 9 isoform 2 in the kidney tubules that causes the excretion of D-glucose and uric acid in urine [21]. Other studies postulated that SGLT-2 inhibitor causes dose-dependent excretion 
of glucose in the urine, leading to more exchange of uric acid in the apical membrane of tubular cells. This causes more release of uric acid from the blood and reduces uric acid concentration in serum $[22,23,24]$. A recent study showed that empagliflozin reduced SUA levels in mice by upregulation of the ATP binding cassette subfamily G member 2 (junior blood group) via the $5^{\prime}$-adenosine monophosphate- (AMP-) activated protein kinase (AMPK)/protein kinase B/cAMPresponse element-binding protein signaling pathway [25]. Moreover, reducing the risk factors of hyperuricemia such as body weight, blood pressure, and insulin resistance by SGLT-2 inhibitors has additional beneficial effects in patients with diabetes.

Our study's main strength was the exclusion of risk factors that can affect SUA levels, such as obesity, hypertension, chronic kidney disease, and uncontrolled diabetes. We also excluded patients taking any drugs that can affect SUA levels. However, our study was limited in that we did not check urine for a complete examination for uric acid, and we did not compare empagliflozin and dapagliflozin for effects on SUA levels.

\section{Conclusions}

SGLT-2 inhibitors have a strong potential to decrease SUA levels in patients with type 2 diabetes. SGLT-2 inhibitors can be administered in patients with diabetes with abnormal SUA levels. Hyperuricemia is one of the potential risk factors for cardiovascular and kidney diseases. Therefore, lowering SUA levels is crucial to prevent morbidity and mortality in patients with type 2 diabetes.

Further studies with larger sample sizes and longer durations should be conducted to illuminate the uric acidlowering effects of SGLT-2 inhibitors.

\section{Data Availability}

The data used to support the findings of this study are available from the corresponding author upon request.

\section{Conflicts of Interest}

The authors declare that they have no conflicts of interest.

\section{Authors' Contributions}

Mazhar Hussain conceived the idea, drafting the article and gave the final approval of manuscript. Abid Hussain designed the study, searched the literature, and wrote the manuscript. Javed Iqbal and Abdul Majid collected clinical, laboratory data and reviewed the manuscript. Lubna Akhtar and Asim Elahi did statistical analysis and interpreted the data with final editing, drafting and approval of manuscript. All members hereby agree to take responsibility of the work and confirm that all questions related to the accuracy and integrity of the research have been properly and thoroughly resolved.

\section{References}

[1] K. Ogurtsova, J. D. da Rocha Fernandes, Y. Huang et al., "IDF Diabetes Atlas: global estimates for the prevalence of diabetes for 2015 and 2040," Diabetes Research and Clinical Practice, vol. 128, pp. 40-50, 2017.

[2] D. R. Whiting, L. Guariguata, C. Weil, and J. Shaw, "IDF Diabetes Atlas: global estimates of the prevalence of diabetes for 2011 and 2030," Diabetes Research and Clinical Practice, vol. 94, no. 3, pp. 311-321, 2011.

[3] Q. Lv, X. F. Meng, F. F. He et al., "High serum uric acid and increased risk of type 2 diabetes: a systemic review and metaanalysis of prospective cohort studies," PLoS One, vol. 8, no. 2, p. e56864, 2013.

[4] S. Kodama, K. Saito, Y. Yachi et al., "Association between serum uric acid and development of type 2 diabetes," Diabetes Care, vol. 32, no. 9, pp. 1737-1742, 2009.

[5] A. Dehghan, M. van Hoek, E. J. Sijbrands, A. Hofman, and J. C. Witteman, "High serum uric acid as a novel risk factor for type 2 diabetes," Diabetes Care, vol. 31, no. 2, pp. 361-362, 2008.

[6] R. Shi, Z. Niu, B. Wu, and F. Hu, "Study on the Risk Factors for Hyperuricaemia and Related Vascular Complications in Patients with Type 2 Diabetes Mellitus," Risk Management and Healthcare Policy, vol. 13, pp. 1661-1675, 2020.

[7] H. Fennoun, N. E. Haraj, S. El Aziz, S. Bensbaa, and A. Chadli, "Risk factors associated with hyperuricemia in patients with diabetes type 2: about 190 cases," Diabetes Research: Open Access, vol. 2, no. 1, pp. 12-16, 2020.

[8] Y. Xu, J. Zhu, L. Gao et al., "Hyperuricemia as an independent predictor of vascular complications and mortality in type 2 diabetes patients: a meta-analysis," PLoS One, vol. 8, no. 10, article e78206, 2013.

[9] Z. Soltani, K. Rasheed, D. R. Kapusta, and E. Reisin, "Potential role of uric acid in metabolic syndrome, hypertension, kidney injury, and cardiovascular diseases: is it time for reappraisal?," Current Hypertension Reports, vol. 15, no. 3, pp. 175-181, 2013.

[10] S. K. Kim, "Interrelationship of uric acid, gout, and metabolic syndrome: focus on hypertension, cardiovascular disease, and insulin resistance," Journal of Rheumatic Diseases, vol. 25, no. 1, pp. 19-27, 2018.

[11] S. Kalra, "Sodium glucose co-transporter-2 (SGLT2) inhibitors: a review of their basic and clinical pharmacology," Diabetes Ther., vol. 5, no. 2, pp. 355-366, 2014.

[12] H. Satoh, "Pleiotropic effects of SGLT2 inhibitors beyond the effect on glycemic control," Diabetology International, vol. 9, no. 4, pp. 212-214, 2018.

[13] C. J. Bailey, "Uric acid and the cardio-renal effects of SGLT2 inhibitors," Diabetes ObesMetab., vol. 21, no. 6, pp. 12911298, 2019.

[14] As part of The Improving Diabetes Steering Committee, A. Ali, S. Bain et al., "SGLT2 inhibitors: cardiovascular benefits beyond HbAlc-translating evidence into practice," Diabetes Therapy, vol. 10, no. 5, pp. 1595-1622, 2019.

[15] M. G. Minze, K. J. Will, B. T. Terrell, R. L. Black, and B. K. Irons, "Benefits of SGLT2 inhibitors beyond glycemic control - a focus on metabolic, cardiovascular and renal outcomes," Current Diabetes Reviews, vol. 14, no. 6, pp. 509-517, 2018.

[16] Z. Hao, X. Huang, H. Shao, and F. Tian, "Effects of dapagliflozin on serum uric acid levels in hospitalized type 2 diabetic patients with inadequate glycemic control: a randomized 
controlled trial," Therapeutics and Clinical Risk Management, vol. Volume 14, pp. 2407-2413, 2018.

[17] H. Ahmadieh and S. Azar, "Effects of sodium glucose cotransporter-2 inhibitors on serum uric acid in type 2 diabetes mellitus," Diabetes Technology \& Therapeutics, vol. 19, no. 9, pp. 507-512, 2017.

[18] Y. Zhao, L. Xu, D. Tian et al., "Effects of sodium-glucose co-transporter 2 (SGLT2) inhibitors on serum uric acid level:Ameta-analysis of randomized controlled trials," Diabetes, Obesity and Metabolism, vol. 20, no. 2, pp. 458-462, 2018.

[19] D. Zhao, H. Liu, and P. Dong, "Empagliflozin reduces blood pressure and uric acid in patients with type 2 diabetes mellitus: a systematic review and meta-analysis," Journal of Human Hypertension, vol. 33, no. 4, pp. 327-339, 2019.

[20] Y. Xin, Y. Guo, Y. Li, Y. Ma, L. Li, and H. Jiang, "Effects of sodium glucose cotransporter-2 inhibitors on serum uric acid in type 2 diabetes mellitus: a systematic review with an indirect comparison meta-analysis," Saudi J Biol Sci., vol. 26, no. 2, pp. 421-426, 2019.

[21] Y. Chino, Y. Samukawa, S. Sakai et al., "SGLT2 inhibitor lowers serum uric acid through alteration of uric acid transport activity in renal tubule by increased glycosuria," Biopharmaceutics \& Drug Disposition, vol. 35, no. 7, pp. 391-404, 2014.

[22] J. B. McGill, “The SGLT2 inhibitor empagliflozin for the treatment of type 2 diabetes mellitus: a bench to bedside review," Diabetes Therapy, vol. 5, no. 1, pp. 43-63, 2014.

[23] C. M. Bruno, G. Pricoco, D. Cantone, E. Marino, and F. Bruno, "Tubular handling of uric acid and factors influencing its renal excretion: a short review," EMJ Nephrology, vol. 4, pp. 92-97, 2016.

[24] A. Novikov, Y. Fu, W. Huang et al., "SGLT2 inhibition and renal urate excretion: role of luminal glucose, GLUT9, and URAT1," American Journal of Physiology. Renal Physiology, vol. 316, no. 1, pp. F173-F185, 2019.

[25] Y. H. Lu, Y. P. Chang, T. Li et al., "Empagliflozin attenuates hyperuricemia by upregulation of ABCG2 via AMPK/AKT/CREB signaling pathway in type 2 diabetic mice," International Journal of Biological Sciences, vol. 16, no. 3, pp. 529$542,2020$. 